\title{
Ammonia sensing using lossy mode resonances in a tapered optical fibre coated with porphyrin-incorporated titanium dioxide
}

\author{
Divya Tiwari*, Kevin Mullaney*, Serhiy Korposh, Stephen W. James*, \\ Seung-Woo Lee ${ }^{\#}$ and Ralph P Tatam* \\ *Engineering Photonics, Cranfield University, Cranfield, Bedfordshire MK43 0AL, UK \\ ${ }^{+}$Advanced Optics Group, Department of Electrical and Electronic Engineering, University of \\ Nottingham, Nottingham, NG7 2RD, UK \\ \#Graduate School of Environmental Engineering, the University of Kitakyushu, 1-1 Hibikino, \\ Kitakyushu 808-0135, Japan
}

\begin{abstract}
The development of an ammonia sensor, formed by the deposition of a functionalised titanium dioxide film onto a tapered optical fibre is presented. The titanium dioxide coating allows the coupling of light from the fundamental core mode to a lossy mode supported by the coating, thus creating lossy mode resonance (LMR) in the transmission spectrum. The porphyrin compound that was used to functionalise the coating was removed from the titanium dioxide coating upon exposure to ammonia, causing a change in the refractive index of the coating and a concomitant shift in the central wavelength of the lossy mode resonance. Concentrations of ammonia as small as $1 \mathrm{ppm}$ was detected with a response time of less than $1 \mathrm{~min}$.
\end{abstract}

Keywords: ammonia sensing, titanium dioxide, molecular imprinting, tapered optical fibre

\section{INTRODUCTION}

Bio-sensing and chemical sensing has been demonstrated using a wide variety of optical fibre based sensing platforms. Often the operation of the sensor relies upon the measurement of analyte induced changes to the optical properties of a functional coating deposited onto the optical fibre. A number of approaches exploit sensitivity to changes in the refractive index of the coating, for example long period gratings ${ }^{1}$, tapered fibres ${ }^{2}$ and surface plasmon resonances ${ }^{3}$.

In recent years there have been a number of reports of the characterisation of devices based upon lossy mode resonances $(\mathrm{LMR})^{4,5}$. LMRs are generated in the transmission spectrum of tapered optical fibre or cladding removed optical fibre coated with thin films where real part of the permittivity of the coating is positive and larger in magnitude than its imaginary part and is larger than that of the surrounding material ${ }^{5}$. Light propagating through the coated optical fibre experiences attenuation maxima at certain wavelengths, dependent on the thickness and refractive index of the coating. This is attributed to the coupling between the core mode and a particular lossy mode of deposited thin film ${ }^{6}$. In recent years, LMRs have been utilised for sensing applications such as refractometers ${ }^{7}$ demonstrating sensitivity higher than that of long period gratings ${ }^{1}$ and $\mathrm{SPR}^{3}$.

In the past decade a new approach for the fabrication of $\mathrm{TiO}_{2}$ thin films based on the liquid phase deposition (LPD) process has attracted a lot of interest due to its cost-effective and simple fabrication process. The deposition of the $\mathrm{TiO}_{2}$ thin films with desired physicochemical properties onto large and complex surfaces at relatively low temperatures, avoiding post fabrication thermal treatment can be achieved readily using this approach. In addition it is relatively easy to functionalise the $\mathrm{TiO}_{2}$ thin film by the addition of the functional compound into the film forming solution.

This paper was published in Proc. SPIE 9916, Sixth European Workshop on Optical Fibre Sensors and is made available with permission of SPIE. One print or electronic copy may be made for personal use only. This paper is issued with: Creative Commons Attribution Non-Commercial No Derivatives License (CC:BY: NC: ND 3.0).

Sixth European Workshop on Optical Fibre Sensors, edited by Elfed Lewis, Proc. of SPIE Vol. 9916, $99161 \mathrm{H} \cdot$ · C $2016 \mathrm{SPIE} \cdot$ CCC code: $0277-786 \mathrm{X} / 16 / \$ 18 \cdot$ doi: $10.1117 / 12.2236926$ 
Additionally, dye sensitised transparent thin $\mathrm{TiO}_{2}$ films have potential in a wide range of applications. For instance tetracationic porphyrin was infused into a $\mathrm{TiO}_{2}$ matrix, prepared by glancing angle physical vapour deposition, to fabricate an optically active and transparent composite film ${ }^{8}$ which was used successfully for the detection of $\mathrm{HCl} \mathrm{gas}{ }^{9}$.

While there has been extensive characterisation of the evolution of the LMR spectrum during the deposition of the coating on a range of optical fibre refractometers, this paper extends the previous research in LMR (discussed above) by utilising LMR for the development of an optical fibre based sensor for the detection of ammonia in water. LMR is achieved in an adiabatic tapered optical fibre by depositing a nano-coating composed of $\mathrm{TiO}_{2}$ with a porphyrin compound as the functional material. The coupling of light from the fundamental core mode to a lossy mode supported by the coating creates attenuation band in the transmission spectrum which is shown to be highly sensitive to the changes in the optical characteristics of the coating.

\section{SENSOR FABRICATION}

\subsection{Tapered optical fibre fabrication}

Adiabatic taper of waist diameter $17 \mu \mathrm{m}$ and $4 \mathrm{~mm}$ taper length was fabricated in a boron-germanium co-doped optical fibre (SMF-28) with cut-off wavelength $620 \mathrm{~nm}$. The output from a Synrad 48-2 $\mathrm{CO}_{2}$ laser with a maximum output power of $25 \mathrm{~W}$ was used to heat the fibre while it was being stretched. The system used to fabricate the tapered optical fibres is described in detail elsewhere [10].

\subsection{Preparation of sensing layers and ammonia sensing}

Boric acid (BA, 61.83g/mol), ammonium hexafluorotitanate (AMPF, Mw: 197.93), Tetrakis(1-methyl-4pyridinio)porphyrin tetra(p-toluenesulfonate) (TMPyP), potassium hydroxide and ammonia $30 \mathrm{wt} \%$ solution were purchased from sigma-Aldrich. Firstly, the section of optical fibre containing the tapered region was fixed in a special holder and the tapered region was immersed into a solution of potassium hydroxide for 20 minutes to treat the surface so that it was terminated with $\mathrm{OH}$ groups. After that, the following solutions were prepared separately in water: $500 \mathrm{mM}$ of $\mathrm{BA}, 100 \mathrm{mM}$ of AMPF and $500 \mathrm{mM}$ of TMPyP. Then, the sensing layer was prepared by incorporating TMPyP into the $\mathrm{TiO}_{2}$ matrix. This was done by adding $(100 \mu \mathrm{l}) \mathrm{TMPyP}$ to the taper holder followed by mixing 1:1 ratio of $(450 \mu 1) 500$ $\mathrm{mM}$ BA and $(450 \mu \mathrm{l}) 100 \mathrm{mM}$ of AMPF. The tapered optical fibre was immersed into the solution for 8.15 hours. Finally, the $\mathrm{TMPyP}^{-\mathrm{TiO}_{2}}$ film was tested for ammonia sensitivity with solutions of various concentrations $(1,10$, and $100 \mathrm{ppm})$ of ammonia in water.

\subsection{Experimental setup}

The experimental setup is shown in Fig 1 (left). The tapered section of the fibre was fixed in a holder with one end connected to light source and other end connected to spectrometer. A tungsten halogen lamp was used as a light source and high resolution spectrometer (Ocean Optics HR4000) was utilised to record the transmission spectrum.

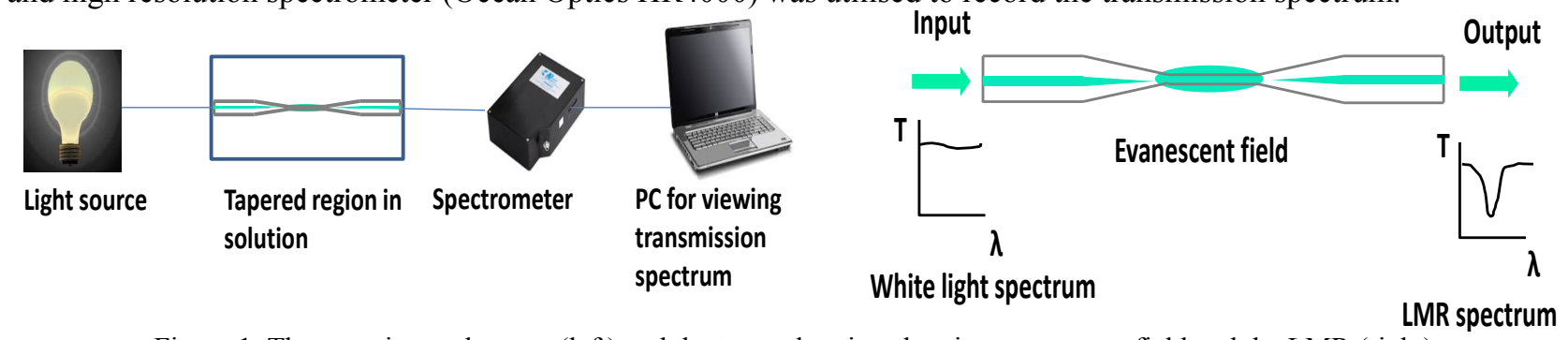

Figure 1. The experimental set-up (left) and the tapered region showing evanescent field and the LMR (right). 


\section{RESULTS AND DISCUSSION}

The nanoscale $\mathrm{TiO}_{2}$ coating was deposited on the tapered region of fibre using the liquid phase deposition method by the chemical reaction between $\mathrm{BA}$ and $\mathrm{AMPF}^{11}$. The transmission spectrum of the $17 \mu \mathrm{m}$ adiabatic tapered fibre was monitored and recorded whilst depositing the $\mathrm{TMPyP}^{-\mathrm{TiO}_{2}}$ coating. Figure 2 presents the evolution of transmission spectrum showing the development of an attenuation band generated by the LMR in response to the increase in optical thickness of coating. The attenuation band appeared in the TS after approximately 7 hours in solution, when the thickness of coating was approximately $70 \mathrm{~nm}$ (measured using SEM). In accordance with previously reported work on LMRs $^{12}$, the attenuation band was found to be highly sensitive to the optical thickness of the coating. As the coating thickness increased, the central resonance wavelength of the attenuation band shifted to higher wavelengths.

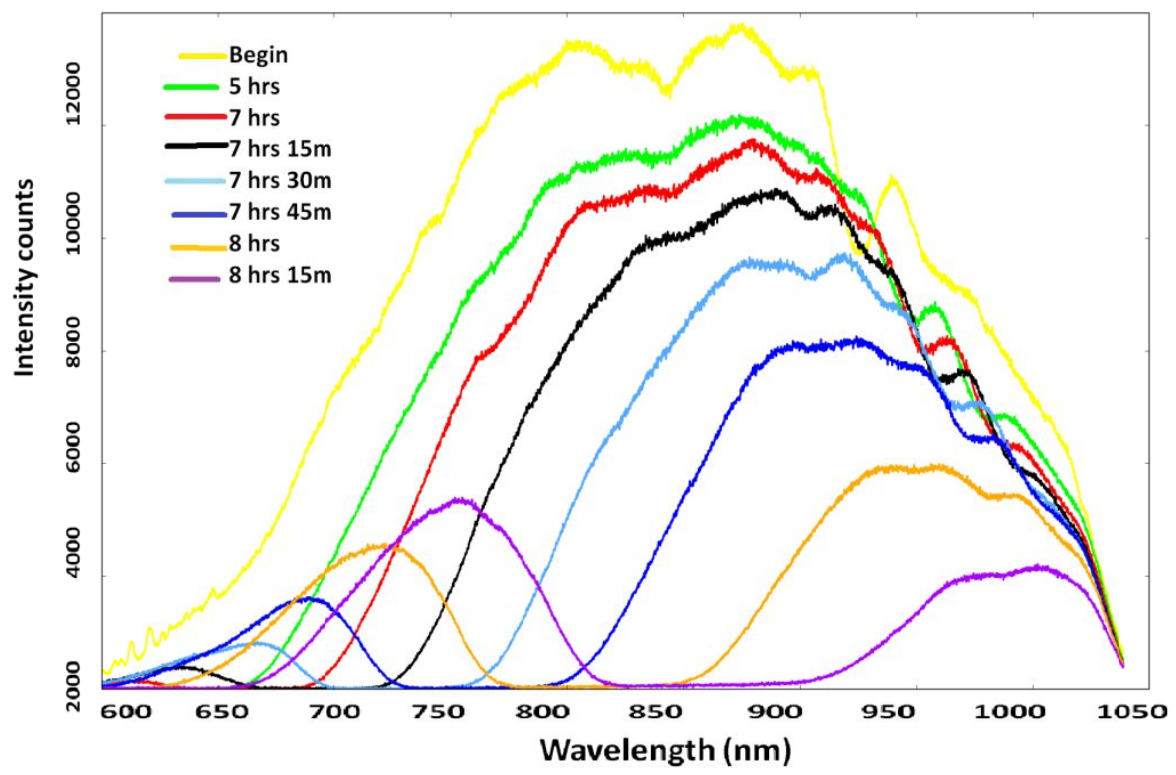

Figure 2. Evolution of the transmission spectrum with the increase in thickness of the $\mathrm{TMPyP}^{\mathrm{TiO}} \mathrm{O}_{2}$ coating.

The coated section of optical fibre was exposed to varying concentrations of ammonia in water and transmission spectrum was monitored for changes in wavelength and intensity. The transmission spectrum was observed in water and then it was monitored while exposing to 1 and $10 \mathrm{ppm}$ concentration of ammonia in water. Upon immersing the coating in $1 \mathrm{ppm}$ ammonia concentration a red-shift of $3 \mathrm{~nm}$ was observed within $30 \mathrm{sec}$ which further enhanced to $3.85 \mathrm{~nm}$ after 3 minutes in solution. This shift in attenuation band to longer wavelengths is continuous and upon exposure to $10 \mathrm{ppm}$ ammonia concentration a red-shift of $4.9 \mathrm{~nm}$ was observed in 1 min. Figure 3 shows a comparison of transmission spectra when immersed in water, and in solution of ammonia of concentration 1 and $10 \mathrm{ppm}$.

The change in transmission spectrum is attributed to the removal of the porphyrin compounded due to the chemical interaction between the ammonia in solution and TMPyP template present in the coating. In contact with ammonia solution, the TMPyP in the sensing layer is desorbed, causing a change in the refractive index of the coating and a change in the central wavelength of the attenuation band. As a result, specific binding sites molecularly imprinted with TMPyP are formed in the $\mathrm{TiO}_{2}$ film coated on the fibre. 


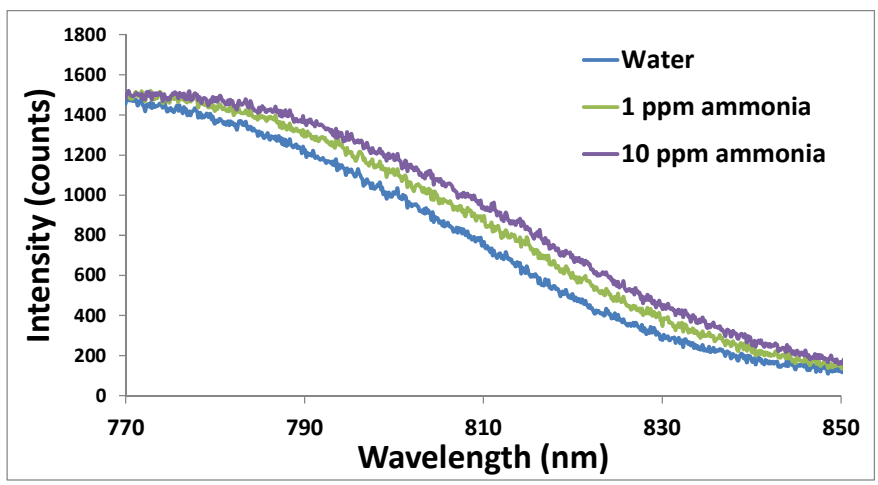

Figure 3. The transmission spectra showing the lower wavelength edge of the LMR attenuation band when immersed in water and in solutions of ammonia of concentrations 1 and $10 \mathrm{ppm}$.

\section{Conclusions}

This work presents an LMR based optical fibre sensor for detection of ammonia in water. The sensor showed high sensitivity to as low as $1 \mathrm{ppm}$ ammonia in water with a response time of less than $1 \mathrm{~min}$. Future work will involve investigation into the re-usability of the sensor by complete desorption of template from the $\mathrm{TiO}_{2}$ coating followed by rebinding of the template.

\section{References}

[1] James, S.W., and Tatam, R. P., "Fiber Optic Sensors with Nano-Structured Coatings," J. Opt. A: Pure Appl. Opt. 8, S430 (2006).

[2] Zibaii, M., Kazemi, A., Latifi, H., Azar, M., Hosseini, S., and Ghezelaiagh, M., "Measuring bacterial growth by refractive index tapered optical fibre biosensor," J. Photochem. Photobiol. B 101, 313-320 (2010).

[3] Zijlstra, P., Paulo P. M., and Orrit, M., "Optical detection of single non-absorbing molecules using the surface plasmon resonance of a gold nanorod", Nat. Nanotechnol 7, 379-382 (2012).

[4] Yang, F. and Sambles, J., R., "Determination of the optical permittivity and thickness of absorbing films using long range modes", J Mod Opt. 44, 1155-1163 (1997).

[5]Villar, I. D., Zamarreño, C. R., Hernaez, M. Arregui, F. J. and Matias I. R., "Lossy mode resonance generation with indium-tin-oxide-coated optical fibers for sensing applications," J. Lightwave Technol. 28, 111-117 (2010)

[6] Marciniak, M., Grzegorzewski, J. and Szustakowski, M.,"Analysis of lossy mode cut-off conditions in planar waveguides with semiconductor guiding layer,” IEEE Proc. J. Optoelectron. 140, 247-252 (1993).

[7] Yang, D. H., Takahara, H., Lee, S. W. and Kunitake T., "Fabrication of glucosesensitive TiO2 ultrathin films by molecular imprinting and selective detection of monosaccharides," Sensors and Actuators B, 130(1),379-385 (2008).

[8] Astillero, J. R. S.V., et al, "Active and Optically Transparent Tetracationic Porphyrin/TiO2 Composite Thin Films," Appl. Mat. and Inter, 2 (3), 712-721 (2010)

[9] Cano, M., Castillero, P., Roales, J., Pedrosa, J. M., Brittle, S., Richardson, T., Barranco, A., "A transparent TMPyP/TiO2 composite thin film as an $\mathrm{HCl}$ sensitive optochemical gas sensor," Sensor Actuat B-Chem, 764-769 (2010).

[10] Mullaney, K., Correia, R., Stains S. E., James, S. W and Tatam., R. P., "Monitoring techniques for the manufacture of tapered optical fibers", Appl.Opt. 54 (28), 8531-8534, (2015).

[11] Lee, M. L., Hung, W. H., "Liquid phase deposition method for growing a titanium dioxide on a gallium arsenide substrate", US 6153539 A, 1999.

[12] Villar, I. D., Hernaez, M., Zamarreno, C. R., Sánchez, P., Valdivielso, C. F., Arregui F. J. and Matias, I. R., "Design rules for lossy mode resonance based sensors," Appl. Opt., 51, 4298-4307 (2012). 


\section{Ammonia sensing using lossy mode resonances in a tapered optical fibre coated with porphyrin-incorporated titanium dioxide}

Tiwari, Divya

Society of Photo-optical Instrumentation Engineers (SPIE)

Divya Tiwari ; Kevin Mullaney ; Serhiy Korposh ; Stephen W. James ; Seung-Woo Lee ; Ralph P. Tatam. Ammonia sensing using lossy mode resonances in a tapered optical fibre coated with porphyrin-incorporated titanium dioxide. Proc. SPIE 9916, Sixth European Workshop on Optical Fibre Sensors. May 30-31, 2016, Limerick, Ireland.

http://dx.doi.org/10.1117/12.2236926

Downloaded from Cranfield Library Services E-Repository 